Check for updates

Cite this: RSC Adv., 2017, 7, 39089

\title{
Visible-light-induced degradation of polybrominated diphenyl ethers with $\mathrm{Agl}-\mathrm{TiO}_{2} \dagger$
}

\begin{abstract}
Ying-Ying Shao, ${ }^{a}$ Wei-Dong Ye, ${ }^{a}$ Chun-Yan Sun, (D) *a Chu-Lin Liu ${ }^{a}$ and Qi Wang ${ }^{b}$
As typical persistent organic pollutants, polybrominated diphenyl ethers (PBDEs) have aroused high environmental concern because of their toxicity and recalcitrant degradation. Here we report the visiblelight-induced degradation of PBDEs with $\mathrm{Agl}-\mathrm{TiO}_{2}(>420 \mathrm{~nm})$. A series of nano-structured $\mathrm{Agl}-\mathrm{TiO}_{2}$ particles were synthesized by a deposition-precipitation method and characterized by SEM, TEM, XRD, XPS, BET and UV-Vis-DRS. The Agl- $\mathrm{TiO}_{2}$ hybrids showed high efficiency for degradation of PBDEs and excellent photostability in cyclic run experiments. The absorbance intensity and absorption ability of $\mathrm{Agl}-\mathrm{TiO}_{2}$ hybrids and the properties of the solvents were found to greatly influence the efficiency of degradation of PBDEs. The $0.2-\mathrm{Agl}-\mathrm{TiO}_{2}$ (the mass ratio of $\mathrm{Ag}$ to $\mathrm{P} 25$ was about $20 \%$ ) in $\mathrm{CH}_{3} \mathrm{OH}$ solution presented the highest reaction rate with $0.29 \pm 0.02 \mathrm{~h}^{-1}$. Most interestingly, mechanistic pathways in the degradation of PBDEs were different under UV and visible light. Unlike the common single-electron transfer in the UV- $\mathrm{TiO}_{2}$ system, the debromination mechanism in the visible light/Agl$\mathrm{TiO}_{2}$ system showed multi-electron transfer. The analysis of products showed that many different lower brominated congeners simultaneously appeared under visible light rather than being stepwise formed under UV. This implies that the special structure of $\mathrm{Agl}-\mathrm{TiO}_{2}$ performs remarkably enhanced electron transfer, multi-electron transfer, and results in high photocatalytic activity. This research may provide a green and efficient method to remove halogenated pollutants by using visible light.
\end{abstract}

Received 27th June 2017 Accepted 28th July 2017

DOI: 10.1039/c7ra07106j

rsc.li/rsc-advances exhibited much higher activity. ${ }^{7}$ Hydrothermal treatment was also introduced as a potential method for the removal of PBDEs. ${ }^{11}$ Recently, we found that PBDEs could undergo efficient debromination under visible light irradiation in the presence of various carboxylate anions..$^{12}$ Nevertheless, the efficiency of degradation still needs to be improved.

Photocatalysis has been proven to be a more effective way to degrade PBEDs. ${ }^{8,13}$ In our previous studies, ${ }^{8}$ PBDEs underwent rapid debromination by $\mathrm{TiO}_{2}$ under UV irradiation, but UV accounts for only $5 \%$ of the solar spectrum. Therefore, in order to effectively utilize solar energy, it is important to realize the photocatalytic degradation of PBDEs under visible light, which is $50 \%$ of the solar spectrum. Silver halides ( $\mathrm{AgX}, \mathrm{X}: \mathrm{Br}, \mathrm{I}$ ) are famous photosensitive materials activated by visible light, and have been widely used as source materials in photochemical reactions. ${ }^{14}$ But one obstacle to their application is instability under irradiation. ${ }^{15}$ When AgI particles are loaded onto the surface of $\mathrm{TiO}_{2}$, the photoelectrons from AgI can be transferred to the conduction band of $\mathrm{TiO}_{2}$ which inhibits the formation of AgI. Thus AgI- $\mathrm{TiO}_{2}$ hybrids perform with high catalytic activity and stability under visible light irradiation for the degradation of organic pollutants. ${ }^{15-24}$ For example, Hu et al. found that $\mathrm{AgI}-\mathrm{TiO}_{2}$ appeared to enhance the efficiency of the degradation of dyes under visible light irradiation. ${ }^{16}$ Nanostructured ${ }^{17,19,21}$ and three dimensional ${ }^{22} \mathrm{AgI-TiO}{ }_{2}$ also showed improved photocatalytic activity. Compared with extensive researches on the
${ }^{a}$ Department of Chemistry, Shaoxing University, Shaoxing 312000, Zhejiang, PR China ${ }^{b}$ School of Environment Sciences and Engineering, Zhejiang Gongshang University, Hangzhou, PR China

$\dagger$ Electronic supplementary information (ESI) available. See DOI: 10.1039/c7ra07106j 
photocatalytic oxidation ability of $\mathrm{AgI}-\mathrm{TiO}_{2}$, the photocatalytic reduction performance of $\mathrm{AgI}-\mathrm{TiO}_{2},{ }^{23}$ especially on the degradation of halogen organic pollutants, is quite rare, which may reveal another important application for $\mathrm{AgI}-\mathrm{TiO}_{2}$. Considering that PBDEs can undergo reduction with $\mathrm{TiO}_{2}$ by UV light irradiation, we suppose that electrons generated from $\mathrm{AgI}-\mathrm{TiO}_{2}$ under visible light might produce an effective reduction of PBDEs under appropriate conditions, which makes the visiblelight-driven photocatalytic degradation of PBDEs possible.

In this work, a series of $\mathrm{AgI}-\mathrm{TiO}_{2}$ photocatalysts were synthesized by deposition-precipitation method. BDE209 (Fig. S1 $\dagger$ ), the major product of PBDEs, ${ }^{1}$ was selected as a target of PBDEs. All prepared AgI-TiO ${ }_{2}$ hybrids exhibited high photocatalytic reduction ability for degradation of BDE209 under visible light irradiation. The superior stability of $\mathrm{AgI}-\mathrm{TiO}_{2}$ was observed in cyclic run experiments. Most interestingly, mechanistic pathways of degradation of PBDEs in a visible light/ AgI-TiO ${ }_{2}$ system showed multi-electron transfer, which was totally different from the common single-electron transfer in the $\mathrm{UV} / \mathrm{TiO}_{2}$ system. To the best of our knowledge, this is the first report on the photocatalytic degradation of PBDEs under visible light irradiation.

\section{Materials and methods}

\subsection{Materials}

BDE209 was obtained from Aldrich Chemical Company (USA). P25 $\left(49 \mathrm{~m}^{2} \mathrm{~g}^{-1}\right)$ was supplied by Degussa. $\mathrm{Al}_{2} \mathrm{O}_{3}$ and $\mathrm{SiO}_{2}$ were purchased from Aladdin Chemistry Co., Ltd. (Shanghai, China). $\mathrm{AgNO}_{3}$ was analytical grade and obtained from Beijing Chemical Reagent Company. All other chemicals were of analytical grade, purchased from Shanghai Chemical Reagent Company and used without further purification. Double distilled water was used throughout the experiments.

\subsection{Synthesis and characterization of photocatalysts}

AgI-TiO ${ }_{2}$ hybrids were prepared as follows: ${ }^{16}$ the suspension was prepared by adding $1 \mathrm{~g}$ of $\mathrm{P} 25$ in $100 \mathrm{~mL}$ of $\mathrm{H}_{2} \mathrm{O}$. After ultrasonic treatment of the suspension for $10 \mathrm{~min}$, a certain amount of KI was then dispersed into the suspension, followed by another $30 \mathrm{~min}$ of ultrasonic treatment. Then, a certain amount of $\mathrm{AgNO}_{3}$ in $\mathrm{NH}_{4} \mathrm{OH}(25 \mathrm{wt} \%)$ solution was added to the suspension and continued under stirring for $5 \mathrm{~h}$. The yellow powder $\left(\mathrm{AgI}-\mathrm{TiO}_{2}\right)$ was filtered, washed with $\mathrm{H}_{2} \mathrm{O}$, and dried in a vacuum oven at $55{ }^{\circ} \mathrm{C}$. The initial mass ratio of $\mathrm{Ag}$ to P25 was about $10 \%$. Thus the as-prepared $\mathrm{AgI}-\mathrm{TiO}_{2}$ was denoted as $0.1-\mathrm{AgI}-\mathrm{TiO}_{2}$. A series of $X-\mathrm{Ag}-\mathrm{TiO}_{2}(X: 0,0.05,0.1,0.15,0.2$, and 0.3) were prepared by adjusting the initial mass ratio of $\mathrm{AgNO}_{3}$ to $\mathrm{P} 25$. $\mathrm{AgI}-\mathrm{SiO}_{2}$ and $\mathrm{AgI}-\mathrm{Al}_{2} \mathrm{O}_{3}$ hybrids were synthesized by a process similar to $\mathrm{AgI}-\mathrm{TiO}_{2}$, except that $\mathrm{TiO}_{2}$ was replaced by $\mathrm{SiO}_{2}$ or $\mathrm{Al}_{2} \mathrm{O}_{3}$. In all cases, the molar ratio of initial $\mathrm{AgNO}_{3}$ to $\mathrm{KI}$ was $1: 1$.

The morphologies of AgI-TiO ${ }_{2}$ were obtained by SEM (Hitachi S4300) and TEM (Philips CM200 FEG TEM at $200 \mathrm{kV}$ ). The XRD patterns of the catalysts were measured on a Rigaku D/Max-2500 diffractometer with $\mathrm{Cu}$ K $\alpha$ radiation $(1.5406 \AA)$. The
XPS spectra of AgI-TiO ${ }_{2}$ were performed by using an ESCA lab 220i-XL spectrometer with an $\mathrm{Al} \mathrm{K} \alpha(1486.6 \mathrm{eV}) \mathrm{X}$-ray source and a charge neutralizer. $\mathrm{N}_{2}$ adsorption-desorption isotherms of AgI-TiO ${ }_{2}$ were obtained by a Quantachrome Autosorb-IQ instrument at $77 \mathrm{~K}$. The specific surface areas of the $\mathrm{AgI}-\mathrm{TiO}_{2}$ samples were obtained by the BET method.

\subsection{BDE209 dehalogenation experiments}

A stock solution of BDE209 $\left(1 \times 10^{-3} \mathrm{~mol} \mathrm{~L}^{-1}\right)$ in THF was diluted to $1 \times 10^{-5} \mathrm{~mol} \mathrm{~L}^{-1}$ with solvents. $10 \mathrm{mg}$ of $\mathrm{AgI}-\mathrm{TiO}_{2}$ was added to $9.95 \mathrm{~mL}$ of BDE209 solution in a Pyrex vessel and $0.05 \mathrm{~mL}$ of isopropanol was added as the hole trapping agent. The total volume of the solution was $10 \mathrm{~mL}$. The reaction solution continued under magnetic stirring during the irradiation experiment. The Pyrex vessel was purged with $\mathrm{N}_{2}$ for 15 min to remove $\mathrm{O}_{2}$ and protected under $\mathrm{N}_{2}$ during the irradiation. The suspensions were stirred for $30 \mathrm{~min}$ in the dark before irradiation to reach adsorption equilibrium. A PLSSXE300 Xe lamp (Beijing Trusttech Co. Ltd.) equipped with a cutoff filter to cut the irradiation below $420 \mathrm{~nm}$ was used as the light source (Fig. S2 $\dagger$ ). At given time intervals, $1 \mathrm{~mL}$ aliquots were used for analysis.

BDE209 was quantified with a SHIMADZU HPLC system (LC-20AT pump and UV/VIS SPD-20A detector) with a DIKMA Platisil ODS C-18 column $(250 \times 4.6 \mathrm{~mm}, 5 \mu \mathrm{m}$ film thickness $)$. The mobile phase was $2 \%$ water in acetonitrile at $1 \mathrm{~mL} \mathrm{~min}{ }^{-1}$ and the detector wavelength was $240 \mathrm{~nm}$. The quantification of BDE209 was obtained from a calibration cure with a BDE-209 standard. Product analysis was performed by $\mathrm{GC}-\mu \mathrm{ECD}$ (Agilent 7890A, Agilent Technologies Co. USA), with a programmable pressure on-column injection port and a DB-5 capillary column $(30 \mathrm{~m} \times 50 \mu \mathrm{m}$, i.d. $\times 0.1 \mu \mathrm{m}$ film thickness $)$. Splitless $10 \mu \mathrm{L}$ injection was performed manually at $300{ }^{\circ} \mathrm{C}$. The carrier gas was helium with a constant flow rate of $1.0 \mathrm{~mL} \mathrm{~min}{ }^{-1}$. The oven temperature was kept at $100{ }^{\circ} \mathrm{C}$ for $2 \mathrm{~min}$, increased to $15{ }^{\circ} \mathrm{C} \min ^{-1}$ up to $230{ }^{\circ} \mathrm{C}$, then increased to $5{ }^{\circ} \mathrm{C} \min ^{-1}$ of $10 \mathrm{~min}$. The standard samples of BDE203, BDE204 and PBDEs (EO5113) were used to identify the degradation products. At given times, $1 \mathrm{~mL}$ aliquots were collected and centrifuged, and filtered to remove the solid catalyst particles before analysis with HPLC and GC- $\mu$ ECD.

In all cases, the degradation of BDE209 was assumed to follow pseudo-first order kinetics, and the rate constant $(k)$ was calculated with the following equation:

$$
\ln \left(C_{0} / C\right)=k t
$$

where $C_{0}$ is the initial concentration of BDE209, $C$ is the concentration of BDE209 at time $(t)$, and $k$ is the pseudo-first order rate constant.

\section{Results and discussion}

\subsection{Characterization of photocatalysts}

As can be seen from Fig. 1 and $\mathrm{S} 3, \dagger$ the morphology and microstructure of AgI-TiO ${ }_{2}$ were obtained by SEM and TEM. The SEM image showed that the shapes of $\mathrm{AgI}-\mathrm{TiO}_{2}$ particles 

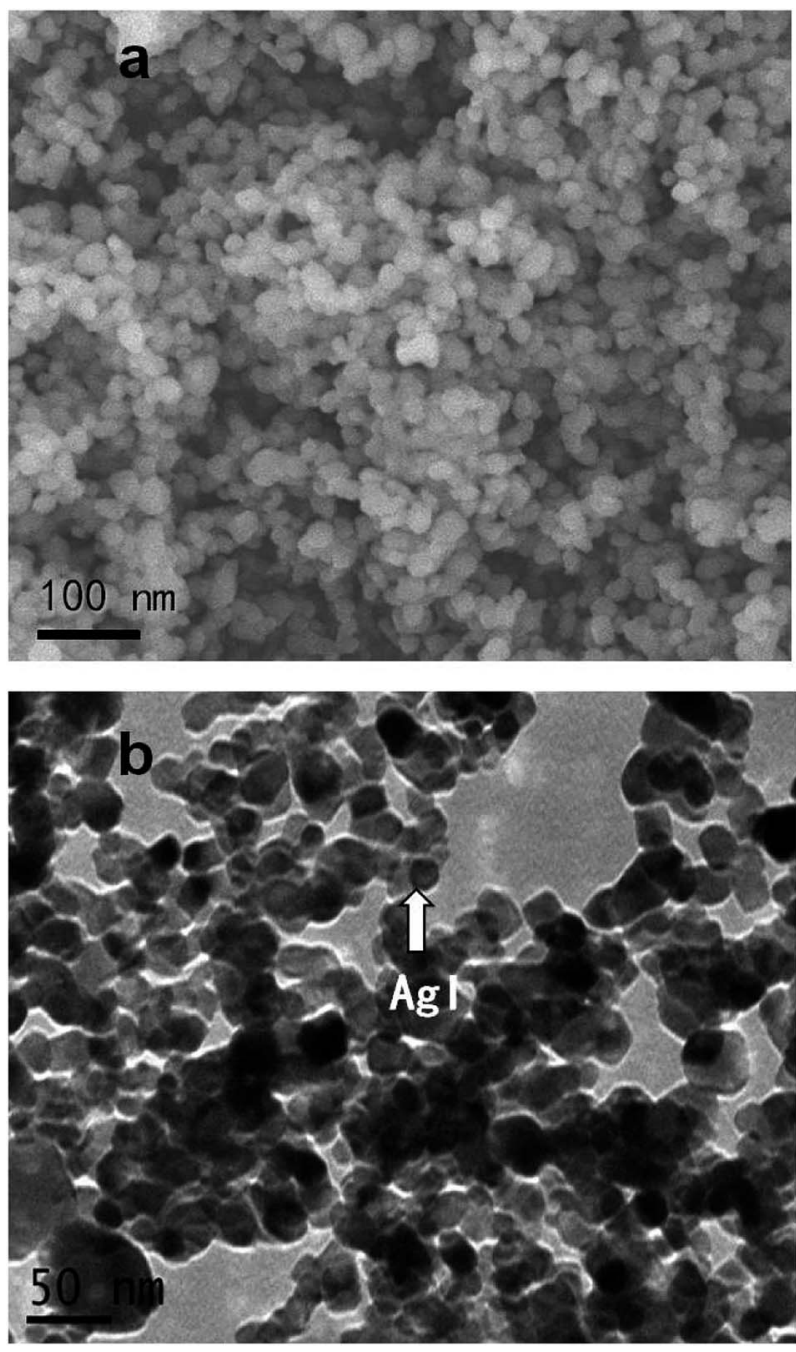

Fig. 1 Images of 0.2-Agl- $\mathrm{TiO}_{2}$ : (a) SEM, (b) TEM.

were irregular spheres. The particle sizes were $20-40 \mathrm{~nm}$ (Fig. 1a). The TEM image displayed the inner structure of the catalyst. AgI displayed nanoparticles with sizes of 10-30 nm dispersed on the surface of $\mathrm{TiO}_{2}$ (Fig. 1b). There was a firm connection between $\mathrm{TiO}_{2}$ and $\mathrm{AgI}$, which helped the transfer of photo-excited electrons between $\mathrm{AgI}$ and $\mathrm{TiO}_{2}$.

The XRD patterns of the $\mathrm{TiO}_{2}$ and $X$-AgI-TiO $2(X: 0.05,0.1$, 0.15, 0.2, and 0.3) are shown in Fig. 2. Without modification of $\mathrm{AgI}, \mathrm{TiO}_{2}$ showed the common anatase and rutile phases of P25. With the addition of AgI, the diffraction peaks attributed to AgI were observed and gradually became stronger. Peaks at $2 \theta$ values of $22.38^{\circ}, 23.70^{\circ}, 25.30^{\circ}, 39.15^{\circ}, 42.70^{\circ}$ and $46.31^{\circ}$ were indexed as the (100), (002), (101), (110), (103) and (112) planes of $\beta$-AgI (JCPDS 09-0374). ${ }^{19}$ In addition, it could be deduced that all the peaks of $\gamma$-AgI overlapped with the peaks of (002), (110), (112) of $\beta$-AgI. According to reported papers, hexagonal $\beta$-AgI and cubic $\gamma$-AgI always coexist at ambient temperature. ${ }^{15}$ No diffraction peaks assigned to $\mathrm{Ag}^{\mathrm{O}}$ were observed in any of the samples. Therefore this indicated that the as-prepared $\mathrm{AgI}-\mathrm{TiO}_{2}$ hybrids were a mixture of $\beta$-AgI and $\gamma$-AgI dispersed on the surface of $\mathrm{TiO}_{2}$ particles.

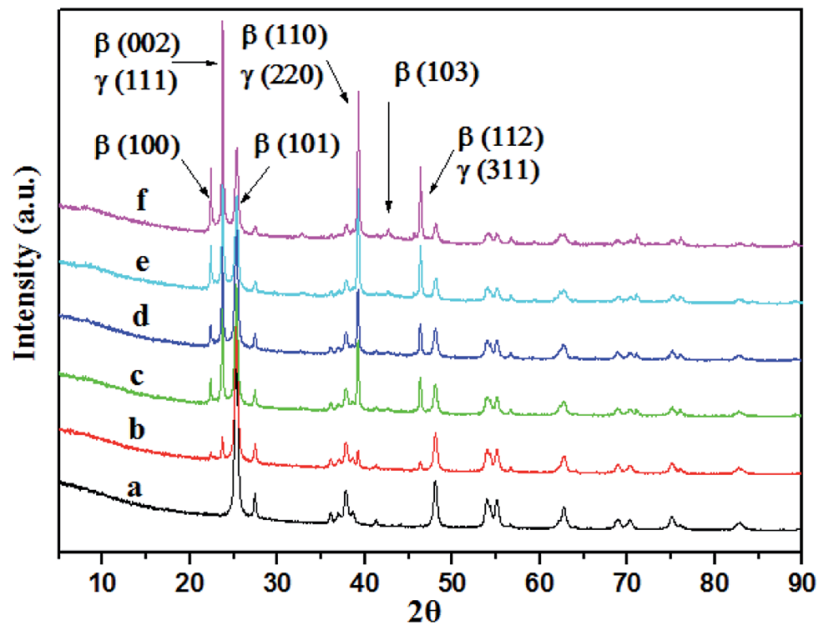

Fig. 2 XRD patterns of $\mathrm{TiO}_{2}$ and $\mathrm{Agl}-\mathrm{TiO}_{2}$. (a) $\mathrm{TiO}_{2}$, (b) 0.05- $\mathrm{Agl}-\mathrm{TiO}_{2}$, (c) $0.1-\mathrm{Agl}-\mathrm{TiO}_{2}$, (d) $0.15-\mathrm{Agl}-\mathrm{TiO}_{2}$, (e) $0.2-\mathrm{Agl}-\mathrm{TiO}_{2}$, (f) $0.3-\mathrm{Agl}-$ $\mathrm{TiO}_{2}$.

XPS measurements were performed to determine the valence state of $\mathrm{Ag}$ and $\mathrm{I}$ in $0.2-\mathrm{AgI}-\mathrm{TiO}_{2}$. As shown in Fig. 3, the binding energy of Ag3 $\mathrm{d}_{5 / 2}$ was $368.5 \mathrm{eV}$, which showed that there was $\mathrm{Ag}^{+}$ in the 0.2-AgI- $\mathrm{TiO}_{2}$ and no $\mathrm{Ag}^{0}$ was formed. Different from previous reports, the value of $\mathrm{Ag} 3 \mathrm{~d}_{5 / 2}$ in $\mathrm{AgI}-\mathrm{TiO}_{2}(368.5 \mathrm{eV})$ was larger than that reported in $\mathrm{AgI} / \mathrm{TiO}_{2}(367.5 \mathrm{eV}),{ }^{16}$ but close to the value in nanostructured $\mathrm{AgI}-\mathrm{TiO}_{2}(368.8 \mathrm{eV}),{ }^{18}$ because the binding energies of $\mathrm{Ag} 3 \mathrm{~d}_{5 / 2}$ are commonly changed according to the size of the Ag nanostructure. ${ }^{18}$ The XPS peak of $13 \mathrm{~d}_{5 / 2}$ appeared at $619.7 \mathrm{eV}$, which was assigned to $\mathrm{I}^{-}$. The peak corresponding to $\mathrm{I}_{2}(620.1 \mathrm{eV})$ was not observed. ${ }^{18}$

The optical properties of the AgI- $\mathrm{TiO}_{2}$ samples were investigated by UV-Vis-DRS. As shown in Fig. 4, without modified AgI particles, $\mathrm{TiO}_{2}$ showed absorption only in the UV region, and the maximum absorbance was at $382 \mathrm{~nm}$. When AgI was combined with $\mathrm{TiO}_{2}$, all the AgI-TiO ${ }_{2}$ samples with different AgI loading amounts exhibited new absorption in the visible light region $(\lambda>400 \mathrm{~nm})$. Moreover, the enhanced absorption was observed with an increasing amount of AgI content and the absorption edges were red shifted to the maximum extent at $450 \mathrm{~nm}$, with the maximum absorbance at $425 \mathrm{~nm}$. Therefore, the absorption of $\mathrm{AgI}-\mathrm{TiO}_{2}$ in the visible light region was attributed to the $\beta$-AgI and $\gamma$-AgI in the hybrids, which have a strong and wide absorption band in the visible light region.

\subsection{Degradation of BDE209 with $\mathrm{AgI}-\mathrm{TiO}_{2}$ under visible light irradiation}

As can be seen from Fig. 5, the direct photolysis of BDE209 did not occur under visible light irradiation. The degradation of BDE209 was scarcely observed in the presence of $\mathrm{TiO}_{2}$ under visible light irradiation. BDE209 also exhibited little degradation when the reactions were carried out with $\mathrm{AgI}-\mathrm{TiO}_{2}$ under dark conditions. However, rapid degradation of BDE209 occurred in the presence of $0.2-\mathrm{AgI}-\mathrm{TiO}_{2}$ under visible light irradiation, and about $70 \%$ of the BDE209 disappeared after $3 \mathrm{~h}$ of irradiation. The kinetics was fitted by a pseudo-first-order 
process, giving a rate constant of $0.29 \pm 0.02 \mathrm{~h}^{-1}$. This indicated that the BDE209 could undergo efficient photocatalytic degradation by $\mathrm{AgI}-\mathrm{TiO}_{2}$ under visible irradiation.

\subsection{Effects of various parameters}

3.3.1 Effects of photocatalyst. Photocatalytic reduction performances of a series of $\mathrm{AgI}-\mathrm{TiO}_{2}$ catalysts were investigated by degradation of BDE209 under visible light irradiation. As shown in Fig. 6, BDE209 underwent efficient degradation by all the synthesized $\mathrm{AgI}-\mathrm{TiO}_{2}$ samples. According to the increase in AgI (mass ratio from 0.05 to 0.3 ), the photoreduction efficiencies of the $\mathrm{AgI}-\mathrm{TiO}_{2}$ hybrids were enhanced, with the kinetic constant increasing from $0.106 \mathrm{~h}^{-1}$ to $0.293 \mathrm{~h}^{-1}$. But the reaction rate decayed to a certain degree when the mass ratio of $\mathrm{Ag}$ reached 0.3 .

In order to reveal the nature of the effects of the photocatalyst, the surfaces of the AgI- $\mathrm{TiO}_{2}$ samples were obtained with $\mathrm{N}_{2}$ adsorption and desorption isotherms and estimated by the BET method. As presented in Table 1, after addition of AgI, the specific surface areas of the catalysts showed a slight decrease. But the loaded amount of AgI had little effect on the specific surface areas of the $\mathrm{AgI}-\mathrm{TiO}_{2}$ samples, which changed from 37.96 to $40.16\left(\mathrm{~m}^{2} \mathrm{~g}^{-1}\right)$. As a heterogeneous catalytic reaction, the photocatalytic reaction occurs primarily on the surface of the catalyst, so the substrate's adsorption ability may
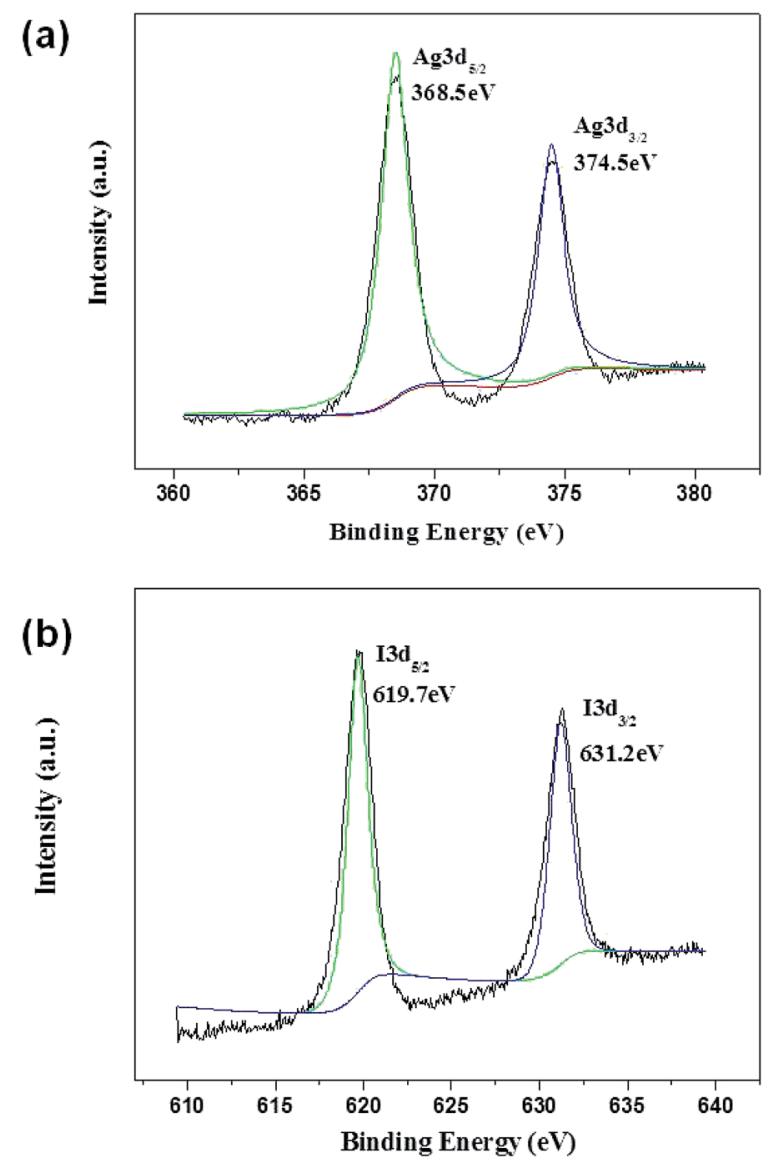

Fig. 3 XPS spectra of Ag3d (a) and I3d (b) for 0.2-Agl- $\mathrm{TiO}_{2}$.

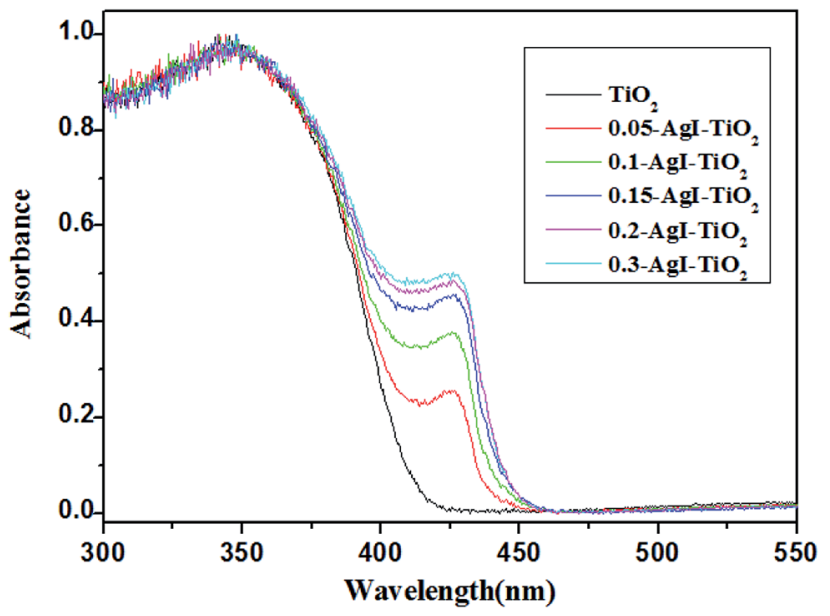

Fig. 4 UV-Vis-DRS of $\mathrm{TiO}_{2}$ and $\mathrm{Agl}-\mathrm{TiO}_{2}$.

have a large effect on the reaction rate. Meanwhile, in the photocatalytic reaction, the absorbance intensity of the catalyst under irradiation is another key factor for the degradation rate. As can be seen from the UV-Vis-DRS (Fig. 4), the absorption intensity of $0.2-\mathrm{AgI}-\mathrm{TiO}_{2}$ was similar to that of $0.3-\mathrm{AgI}-\mathrm{TiO}_{2}$. But the amount of BDE209 absorbed on the surface of $0.2-\mathrm{AgI}-\mathrm{TiO}_{2}$ was more than that of $0.3-\mathrm{AgI}-\mathrm{TiO}_{2}$. Therefore, among all the hybrids, the degradation of BDE209 with 0.2-AgI-TiO ${ }_{2}$ was fastest, at nearly 3 times that of $0.05-\mathrm{AgI}-\mathrm{TiO}_{2}$.

3.3.2 Effects of solvents. In order to investigate the effect of the solvent, the reactions were carried out in eight different solvents (Table $\mathrm{S} 1 \dagger$ ). In general, the heterogeneous photocatalysis reaction occurs primarily on the surface of the catalyst. The adsorption ability of the catalyst largely influences the reduction kinetics. In toluene, acetone, hexane, THF, and DMSO solutions, where the ability of the catalyst to adsorb BDE209 was very weak, degradation scarcely occurred. However, adsorption is not the only key factor controlling the photocatalytic degradation of BDE209. The amount of BDE209 adsorbed in $\mathrm{CH}_{3} \mathrm{CN}$ (20.4\%) was lower than in the DMF (4.1\%),

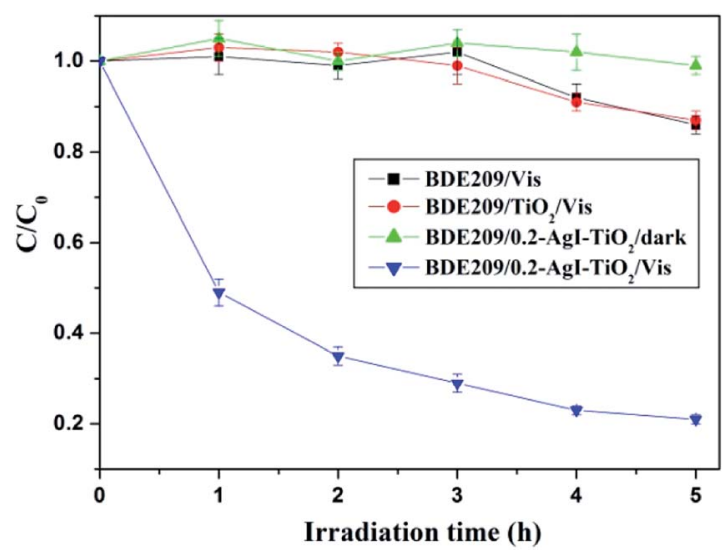

Fig. 5 Temporal curves of the photodegradation of BDE209 under different conditions. BDE209: $1.0 \times 10^{-5} \mathrm{~mol} \mathrm{~L}^{-1}$, catalyst: $1 \mathrm{mg} \mathrm{mL}^{-1}$, solvent: $\mathrm{CH}_{3} \mathrm{OH}$, wavelength $>420 \mathrm{~nm}$, anoxic condition. 


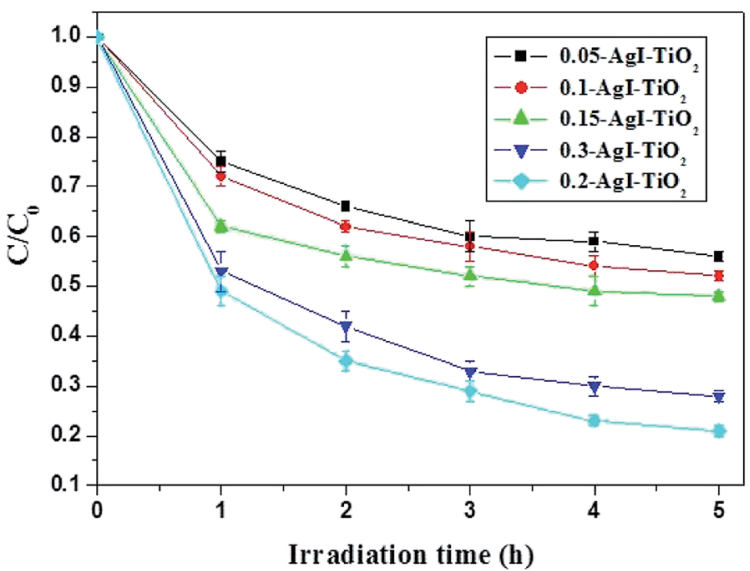

Fig. 6 Temporal curves of the photodegradation of BDE209 under different mass ratios of $\mathrm{Agl} / \mathrm{TiO}_{2}$ under visible irradiation. BDE209: $1.0 \times 10^{-5} \mathrm{~mol} \mathrm{~L}^{-1}, \mathrm{Agl}-\mathrm{TiO}_{2}: 1 \mathrm{mg} \mathrm{mL}^{-1}$, solvent: $\mathrm{CH}_{3} \mathrm{OH}$, wavelength $>420 \mathrm{~nm}$, anoxic condition.

but the degradation rate of BDE209 in DMF (56.6\%) was higher than that in $\mathrm{CH}_{3} \mathrm{CN}$ (33.6\%). Among all eight organic solvents, the degradation rate of $\mathrm{BDE} 209$ in $\mathrm{CH}_{3} \mathrm{OH}(71.1 \%)$ was highest, because $\mathrm{CH}_{3} \mathrm{OH}$ was a good VB hole scavenger and hydrogen donor in the reaction. Meanwhile, the amount of BDE209 adsorbed in $\mathrm{CH}_{3} \mathrm{OH}(30 \%)$ is a maximum among all eight solvents. With the poor performances of solvents either on adsorption or on the hydrogen donor, degradation of BDE209 was scarcely observed. This implies that the solvents participate in the visible-light-induced degradation of PBDEs. When a small amount of water was added to the dispersion, the degradation rate of BDE209 decreased (Fig. S4†). Because BDE209 is very hydrophobic, the electron transformation channels from the conduction bands of $\mathrm{AgI}-\mathrm{TiO}_{2}$ to BDE209 would be held back by water. That places some impediments on the treatment of BDE209. Preloading BDE209 onto the surface of the catalysts can make the hydrophobic BDE209 undergo photocatalytic degradation in water. ${ }^{13}$ Due to the complexity of photocatalytic systems, many mechanistic details, including the effect of solvents on the electron transfer and the formation of the reducing species, need to be researched further.

3.3.3 Photostability of photocatalyst. Photostability is an important factor for a photocatalyst in practical application. So recycling experiments were performed to test the photostability.

Although AgI can be easily photo-decomposed into $\mathrm{Ag}^{0}$, $\mathrm{AgI}-\mathrm{TiO}_{2}$ displayed high photostability in the run experiments. As shown in Fig. 7, after four cycles, $\mathrm{AgI}-\mathrm{TiO}_{2}$ still showed high photoreduction ability for the degradation of BDE209. The reaction rates were not obviously decreased in the run experiments. The photo-generated electrons from AgI could be transformed to the conduction bands of $\mathrm{TiO}_{2}$ not $\mathrm{Ag}^{+}$, so the stability of AgI was greatly enhanced in the $\mathrm{AgI}-\mathrm{TiO}_{2}$ hybrids. ${ }^{16}$

\subsection{New degradation pattern under visible light irradiation}

In general, the degradation of PBDEs is caused by the loss of bromine atoms by reduction. It is known that the bioaccumulation and toxicity of PBDEs depend on the number of
Table $1 S_{B E T}\left(\mathrm{~m}^{2} \mathrm{~g}^{-1}\right)$, absorption amount and estimated pseudo-firstorder kinetic constant $(k)$ for the degradation of BDE209 with Agl$\mathrm{TiO}_{2}$

\begin{tabular}{lllll}
\hline Catalyst & $\begin{array}{l}\text { AgI/TiO } \\
\text { mass ratio }\end{array}$ & $\begin{array}{l}S_{\mathrm{BET}} \\
\left(\mathrm{m}^{2} \mathrm{~g}^{-1}\right)\end{array}$ & $\begin{array}{l}\text { Absorption } \\
\text { amount }(\%)\end{array}$ & $\begin{array}{l}\text { Kinetic } \\
\text { constant } k\left(\mathrm{~h}^{-1}\right)\end{array}$ \\
\hline $\mathrm{TiO}_{2}$ & & 42.2 & 31.0 & \\
0.05-AgI-TiO $_{2}$ & 0.05 & 38.4 & 28.8 & $0.106 \pm 0.01$ \\
0.1-AgI-TiO $_{2}$ & 0.10 & 39.2 & 29.0 & $0.120 \pm 0.02$ \\
$0.15-\mathrm{AgI}-\mathrm{TiO}_{2}$ & 0.15 & 39.9 & 29.8 & $0.127 \pm 0.03$ \\
$0.2-\mathrm{AgI}-\mathrm{TiO}_{2}$ & 0.20 & 40.2 & 30.2 & $0.293 \pm 0.02$ \\
$0.3-\mathrm{AgI}-\mathrm{TiO}_{2}$ & 0.30 & 38.1 & 28.4 & $0.238 \pm 0.02$ \\
\hline
\end{tabular}

bromine atoms and their substituted positions. So it is very important to reveal the debromination pattern by product analysis. The less brominated products are more resistant to reduction than the more brominated congeners, so BDE209 commonly prefers to undergo debromination in a stepwise way. For example, in our previous study of the $\mathrm{TiO}_{2} / \mathrm{UV}$ system, ${ }^{8}$ the degradation of BDE209 showed a stepwise manner via singleelectron transfer by $\mathrm{TiO}_{2}$. At the beginning of the irradiation, only 9Br-BDEs were observed and the amount of 9Br-BDEs increased. Then 9Br-BDEs disappeared and 8Br-BDEs were formed. In this stepwise method of degrading, the lower bromine products formed only after the higher bromine parent congeners had disappeared. However, it is most interesting that the debromination pattern in the degradation of PBDEs in visible light is totally different to that in UV.

The degradation products were identified and quantified by HPLC and GC- $\mu$ ECD with the relative retention times of chemical standards and the orders of GC elution obtained from the literature (8, 12 and 13) (Fig. 8 and S5†). After visible light irradiation, BDE209 was reduced to its lower bromine congeners by $\mathrm{AgI}-\mathrm{TiO}_{2}$. Before the irradiation, only the dominant GC peak of BDE209 (10Br) was observed. However, it was interesting that 6-9 Br congeners appeared simultaneously after $5 \mathrm{~h}$ of irradiation. With further prolonged irradiation time to $24 \mathrm{~h}$, the lower bromine congeners were observed and their amounts gradually increased. The product analysis showed that a large

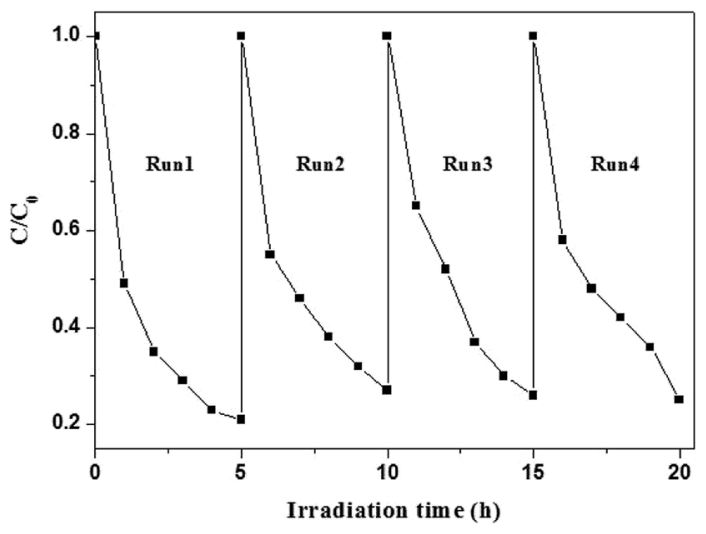

Fig. 7 Cycling runs in the degradation of $\mathrm{BDE} 209$ with $\mathrm{Agl}-\mathrm{TiO}_{2}$ under visible irradiation. BDE209: $1.0 \times 10^{-5} \mathrm{~mol} \mathrm{~L}^{-1}, 0.2-\mathrm{Agl}_{-} \mathrm{TiO}_{2}$ : $1 \mathrm{mg} \mathrm{mL}{ }^{-1}$, solvent: $\mathrm{CH}_{3} \mathrm{OH}$, wavelength $>420 \mathrm{~nm}$, anoxic condition. 
amount of the different lower bromine congeners were formed simultaneously (Fig. 8). This implied that the degradation of BDE209 by $\mathrm{AgI}-\mathrm{TiO}_{2}$ did not follow the stepwise mechanism via single electron transfer. The simultaneous generation of different brominated congeners required more electrons to be transferred at the same time from AgI-TiO ${ }_{2}$ hybrids to BDE209. Therefore, a distinctive multi-electron transfer mechanism occurred in the AgI-TiO $/$ /visible system. A similar debromination mechanism with multi-electron transfer was also reported in the degradation of BDE209 by graphene/ $/ \mathrm{TiO}_{2} \cdot{ }^{25}$ This special debromination pattern via multi-electron transfer may skip some reduction steps, avoiding the formation of some more toxic, more refractory intermediates.

\subsection{The mechanism of reaction}

UV-Vis-DRS showed that photocatalytic activity under visible light irradiation was attributed to AgI. With loading of AgI particles, a new absorption in the visible light region was observed and the absorption intensity became stronger with an increase in the amount of AgI. This was also proved by control experiments with $\mathrm{AgI}-\mathrm{SiO}_{2}$ and $\mathrm{Ag}-\mathrm{Al}_{2} \mathrm{O}_{3}$. As shown in Fig. $\mathrm{S} 6, \uparrow$ a certain extent of degradation of BDE209 was observed in the presence of $\mathrm{AgI}-\mathrm{SiO}_{2}$ or $\mathrm{Ag}-\mathrm{Al}_{2} \mathrm{O}_{3}$ under visible light irradiation, but the reaction was almost stopped when about 40\% BDE209 had degraded. However, about $80 \%$ of BDE209 was degraded with $\mathrm{AgI}-\mathrm{TiO}_{2}$ under the same experimental conditions. This indicated that in the $\mathrm{AgI}-\mathrm{SiO}_{2}$ or $\mathrm{Ag}-\mathrm{Al}_{2} \mathrm{O}_{3}$ system, AgI was excited by visible light and photoelectrons from AgI could transfer to BDE209, which resulted in the degradation of BDE209. But AgI was soon decomposed and the reaction stopped due to the instability of AgI under the irradiation. However, when the AgI particles were loaded onto the surface of $\mathrm{TiO}_{2}$, the photoelectrons from AgI could be transferred via the conduction band of $\mathrm{TiO}_{2}$ and inhibited the decomposition of AgI. Therefore AgI- $\mathrm{TiO}_{2}$ hybrids showed a higher catalytic efficiency than $\mathrm{AgI}-\mathrm{SiO}_{2}$ and $\mathrm{Ag}-\mathrm{Al}_{2} \mathrm{O}_{3}$. Unlike the instability of AgI, AgI-TiO ${ }_{2}$ hybrids performed with good stability for the degradation of BDE209 under visible light irradiation, which was proved by the cyclic run experiments. An oxygen control

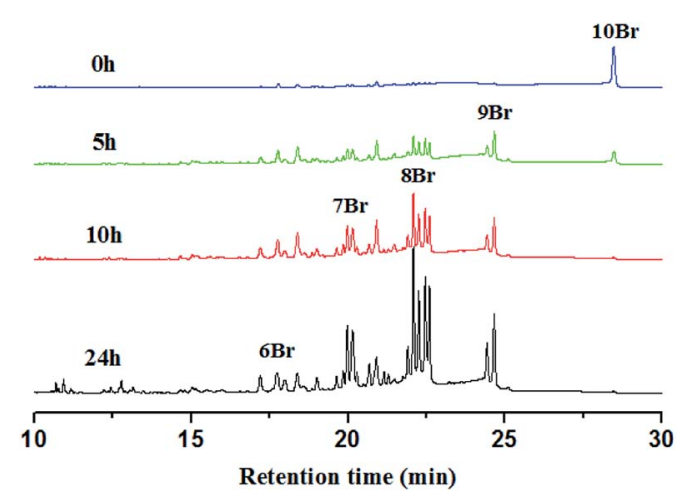

Fig. 8 GC- $\mu E C D$ chromatograms of degradation products of BDE209 with $\mathrm{Agl}-\mathrm{TiO}_{2}$ with different irradiation times. Reaction conditions: BDE209: $1.0 \times 10^{-5} \mathrm{~mol} \mathrm{~L}^{-1} ; 0.2-\mathrm{Agl}_{-} \mathrm{TiO}_{2}: 1 \mathrm{mg} \mathrm{mL}{ }^{-1}$, solvent: $\mathrm{CH}_{3} \mathrm{OH}$, wavelength $>420 \mathrm{~nm}$, anoxic condition.

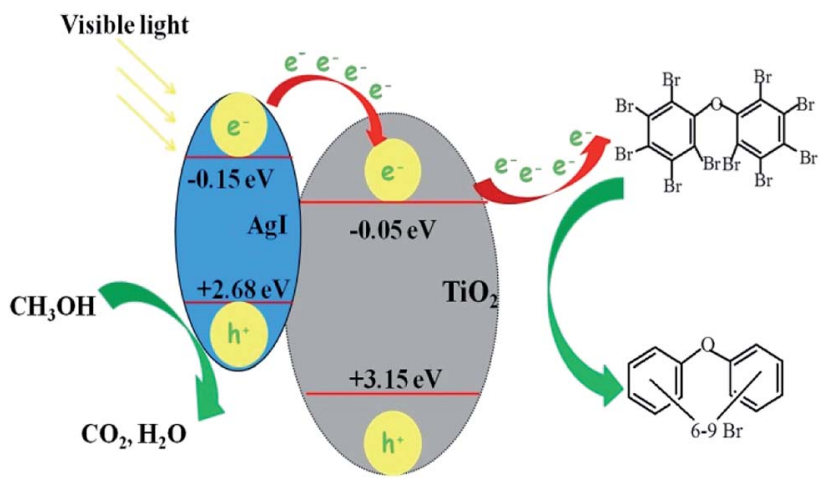

Fig. 9 The mechanism of degradation of $\mathrm{BDE} 209$ in $\mathrm{CH}_{3} \mathrm{OH}$ with $\mathrm{Agl}-\mathrm{TiO}_{2}$ under visible light irradiation. ${ }^{23}$

experiment showed that the degradation of BDE209 was largely prohibited in presence of $\mathrm{O}_{2}$ (Fig. S6 $\dagger$ ). This indicates that BDE209 prefers to be reduced by the electrons in the conduction bands of $\mathrm{AgI}-\mathrm{TiO}_{2}$ and is resistant to oxidization by VB holes or reactive oxygen species in the presence of $\mathrm{O}_{2}$.

Based on the above experiments, a possible reaction mechanism is proposed in Fig. 9. Under visible light irradiation, AgI was excited and then photogenerated $\mathrm{e}-\mathrm{h}^{+}$pairs from AgI were separated. In the anaerobic condition with $\mathrm{N}_{2}$, the VB holes were scavenged by $\mathrm{CH}_{3} \mathrm{OH}$ and the photogenerated electrons of AgI accumulated and preferred to transfer to the conduction bands of $\mathrm{TiO}_{2}$, which resulted in the inhibition of the recombination of $\mathrm{e}-\mathrm{h}^{+}$pairs and the more efficient separation of $\mathrm{e}-\mathrm{h}^{+}$ pairs. Subsequently, the enhanced electron transfer, multielectron transfer, occurred between the BDE209 and $\mathrm{AgI}-\mathrm{TiO}_{2}$. BDE209 received more than one electron and was degraded to different lower bromo congeners at same time. Because the photo-generated electrons from AgI were transformed to the conduction bands of $\mathrm{TiO}_{2}$ not $\mathrm{Ag}^{+}, \mathrm{AgI}-\mathrm{TiO}_{2}$ showed excellent photostability in the run experiments.

\section{Conclusions}

A series of nanostructured $\mathrm{AgI}-\mathrm{TiO}_{2}$ particles were synthesized by a deposition-precipitation method and characterized by SEM, TEM, XRD, XPS, BET and UV-Vis-DRS. The AgI-TiO hybrids showed high photo-reduction capability and good stability for degradation of BDE209 under visible light irradiation. The absorbance intensity and absorption ability of the $\mathrm{AgI}-\mathrm{TiO}_{2}$ hybrids and the properties of solvents greatly influenced the efficiency of the degradation of PBDEs. The 0.2-AgI- $\mathrm{TiO}_{2}$ hybrid in $\mathrm{CH}_{3} \mathrm{OH}$ solution presented the highest reaction rate at $0.29 \pm 0.02 \mathrm{~h}^{-1}$. Mechanistic pathways in degradation of PBDEs were different under UV and visible light. The debromination in the visible-light-induced $\mathrm{AgI}^{-\mathrm{TiO}_{2}}$ system showed a multi-electron transfer mechanism, which is different from the single-electron transfer in the $\mathrm{UV}^{-\mathrm{TiO}_{2}}$ system. The AgI-TiO ${ }_{2}$ hybrid showed an enhanced electron transfer in the photocatalytic process and resulted in high activity in photocatalytic reduction. This research may provide 
a green and efficient method of removing halogenated pollutants.

\section{Acknowledgements}

The generous financial support by the National Science Foundation of China (No. 21107073 and 21477080) and the China Scholarship Council is gratefully acknowledged.

\section{References}

1 L. S. Brinbaum and D. F. Staskal, Environ. Health Perspect., 2004, 112, 9-17.

2 P. P. He, C. S. He, Q. Liu and Y. Mu, $R S C A d v ., 2017,7,27214-$ 27223.

3 B. X. Mai, S. J. Chen, X. J. Luo, L. G. Chen, Q. S. Yang, G. Sheng, P. Peng, J. Fu and E. Zeng, Environ. Sci. Technol., 2005, 39, 3521-3527.

4 H. M. Stapleton, B. Brazil, R. D. Holbrook, C. L. Mitchelmore, R. Benedict, A. Konstantinov and D. Potter, Environ. Sci. Technol., 2006, 40, 4653-4658.

5 J. Bezares-Cruz, C. T. Jafvert and I. Hua, Environ. Sci. Technol., 2004, 38, 4149-4156.

6 Y. S. Keum and Q. X. Li, Environ. Sci. Technol., 2005, 39, 22802286.

7 A. Li, C. Tai, Z. S. Zhao, Y. W. Wang, Q. H. Zhang, G. B. Jiang and J. T. Hu, Environ. Sci. Technol., 2007, 41, 6841-6846.

8 C. Y. Sun, D. Zhao, C. C. Chen, W. H. Ma and J. C. Zhao, Environ. Sci. Technol., 2009, 43, 157-162.

9 X. Li, J. Huang, G. Yu and S. B. Deng, Chemosphere, 2010, 78, 752-759.

10 A. Z. Huang, N. Wang, M. Lei, L. H. Zhu, Y. Y. Zhang, Z. F. Lin, D. Q. Yin and H. Q. Tang, Environ. Sci. Technol., 2013, 47, 518-525.
11 K. S. Nose, S. J. Hashimoto, S. Takahashi, Y. K. Noma and S. I. Sakai, Chemosphere, 2007, 68, 120-125.

12 C. Y. Sun, W. Chang, W. H. Ma, C. C. Chen and J. C. Zhao, Environ. Sci. Technol., 2013, 47, 2370-2377.

13 C. Y. Sun, J. C. Zhao, H. W. Ji, W. H. Ma and C. C. Chen, Chemosphere, 2012, 89, 420-425.

14 C. Hu, T. W. Peng, X. X. Hu, Y. L. Nie, X. F. Zhou, J. H. Qu and H. He, J. Am. Chem. Soc., 2010, 132, 857-862.

15 A. C. Zhang, L. X. Zhang, X. Z. Chen, Q. F. Zhu, Z. C. Liu and J. Xiang, Appl. Surf. Sci., 2017, 392, 1107-1116.

$16 \mathrm{C} . \mathrm{Hu}, \mathrm{X} . \mathrm{Hu}, \mathrm{L}$. Wang, J. H. Qu and A. Wang, Environ. Sci. Technol., 2006, 40, 7903-7907.

17 D. D. Yu, J. Bai, H. O. Liang, J. Z. Wang and C. P. Li, Appl. Surf. Sci., 2015, 349, 241-250.

18 D. Wu and M. Long, ACS Appl. Mater. Interfaces, 2011, 3, 4770-4774.

19 Y. Li, H. Zhang, Z. Guo, J. Han, X. Zhao, Q. Zhao and S. Kim, Langmuir, 2008, 24, 8351-8357.

20 S. Song, F. Hong, Z. He, Q. Cai and J. Chen, J. Colloid Interface Sci., 2012, 378, 159-166.

21 J. G. Yu, G. P. Dai and B. B. Huang, J. Phys. Chem. C, 2009, 113, 16394-16401.

22 C. H. An, W. Jiang, J. Z. Wang, S. T. Wang, Z. H. Ma and Y. P. Li, Dalton Trans., 2013, 42, 8796-8801.

23 Q. Wang, X. D. Shi, J. J. Xu, J. C. Crittenden, E. Q. Liu, Y. Zhang and Y. Q. Cong, J. Hazard. Mater., 2016, 307, 213220.

24 L. Yang, M. G. Gao, B. Dai, X. H. Guo, Z. Y. Liu and B. H. Peng, Appl. Surf. Sci., 2016, 386, 337-344.

25 M. Lei, N. Wang, L. H. Zhu, C. S. Xie and H. Q. Tang, Chem. Eng. J., 2014, 241, 207-215. 